\title{
Measurement of Wakefield Suppression in a Detuned X-Band Accelerator Structure
}

\author{
C. Adolphsen, K. Bane, T. Higo*, K. Kubo*, R. Miller, R. Ruth, K. Thompson, and J. Wang \\ Stanford Linear Accelerator Center, Stanford University, Stanford, California 94309
}

\begin{abstract}
Research is underway at SLAC to develop accelerator structures for a next generation linear collider. A fullscale prototype X-band structure has been built in which the dipole mode frequencies were detuned to suppress the long-range transverse wakefield by about two orders of magnitude. To verify that the detuning works as expected, a facility to measure the long-range wakefield, called the Accelerator Structure SETup, or ASSET, was constructed in the SLAC Linear Collider (SLC). This paper presents the results from the measurement of the prototype X-band structure with this facility.
\end{abstract}

The designs being considered at SLAC for the Next Linear Collider (NLC) employ multi-bunch operation in a high acceleration gradient X-band linac [1]. One problem that has to be addressed in such an approach is the blowup of the transverse motion of the bunch train due to wakefield forces. That is, the long-range transverse wakefield generated by the bunch train in the X-band accelerator structures resonantly amplifies the betatron motion of the train. The lowest band of dipole modes in the structures are the most harmful in this regard. If the structures in an NLC linac were built with identical cells, so essentially only the synchronous mode (phase velocity $=c$ ) in this band would be excited, the blowup would be enormous. To reduce the wakefield to a manageable level, a structure detuning strategy has been developed that exploits the wakefield decoherence that occurs when many modes in the lowest band are excited. In particular, the cells of the structure are designed with a smoothly varying geometry that maintains a constant accelerating mode frequency $(11.4 \mathrm{GHz})$ along the structure while producing a Gaussian distribution in frequency of the product of the dipole mode density and the mode coupling strength to the beam. As a result, the integrated dipole wakefield, which evolves in time essentially as the inverse Fourier transform of this product, also falls off in a Gaussian manner.

A full-scale ( $1.8 \mathrm{~m}$ long) prototype X-band structure has been built with this detuning strategy and successfully operated at high power [2]. The structure parameters were chosen to reduce the wakefield generated by one bunch by about two orders of magnitude within the 1.4 ns period between bunches in the NLC design. The geometry of its 206 cells are such that if one periodic structure were made from each cell type, the resulting density, $\mathrm{dn} / \mathrm{d} v$, of the lowest-band synchronous mode frequencies, $v$, of the 206 structures would be Gaussian with $\sigma_{v} / v=2.5 \%$, a central frequency of $15.1 \mathrm{GHz}$ and $a \pm 2 \sigma_{v}$ truncation of its tails. To achieve this distribution, the cells vary in iris radius (5.72 to $3.90 \mathrm{~mm}$ ), iris thickness (1 to $2 \mathrm{~mm}$ ), and cavity radius (11.44 to $10.68 \mathrm{~mm}$ ) along the structure. Although the iris thickness variation is not required to detune the lowest band of dipole modes, it was added to further detune the smaller contributions from the higher bands of dipole modes [3].

The variation in the cell geometry is expected to yield a dipole mode density, which when weighted by the mode coupling strength to the beam, has a distribution similar to that described above for the synchronous modes of the associated periodic structures, except that its tails go smoothly to zero instead of being truncated. This result is based on an equivalent circuit model of the structure that characterizes each cell as a two mode oscillator that is weakly coupled to its neighbors [4]. However, the wakefield predicted by this model, which is shown below, does not differ greatly from that computed by assuming that the structure behaves as a set of uncoupled oscillators with frequencies equal to those of the synchronous modes of the associated periodic structures.

One shortcoming of the detuning scheme is that at times comparable to the length of the NLC bunch train, which is nominally $126 \mathrm{~ns}$, the wakefield is expected to have recohered to several percent of its initial strength as a result of the discreteness of the mode frequencies. Although this level is about an order-of-magnitude larger than can be tolerated, a wakefield reduction of this size can be achieved by using four structure types with interleaved mode frequencies, or by weakly damping the modes [5].

With the large wakefield suppression required, and the approximate nature of the predictions, a search was made for an experimental means to verify the effect of the detuning on the long-range transverse wakefield. During this process, it was realized that the SLC was well suited as a test bed because its electron and positron bunches can be independently injected into the main linac to serve as drive and witness bunches for wakefield measurements [6]. Also, the few hundred $\mu \mathrm{m}$ transverse sizes of the bunches at injection are small in comparison to the structure iris diameters so the bunch trajectories can be readily varied within the structures for such measurements. Consequently, the Accelerator Structure SETup, or ASSET [7], was constructed near the beginnin' of the main linac in the

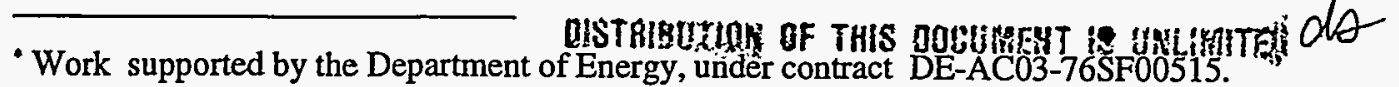


SLC for the purpose of measuring the long-range wakefield generated in a single X-band structure. The remainder of this paper describes ASSET, the wakefield measurement methodology, and the results from the measurement of the prototype X-band structure in the first use of this facility.

Figure 1 shows a plan view sketch of ASSET with the structure installed. During the measurements, the structure was maintained at its nominal operating temperature, and its input and output if couplers were terminated with matched loads.

For the transverse wakefield measurements, the positron bunch served as the drive bunch, and was extracted from the South Damping Ring and injected into the main linac via the South-Ring-To-Linac (SRTL) transport line. In the linac, the bunch passed through the X-band structure and was then steered into a dump. The magnet used for this purpose is also the first bend of a chicane which transported electrons back onto the linac axis. The electron bunch served as the witness bunch, and was extracted from the North Damping Ring at a later time $(\equiv t)$ and injected on-axis into the linac via the North-Ring-To-Linac (NRTL) transport line. In traversing the test structure, the witness bunch was deflected by the wakefield generated by the drive bunch. The witness bunch then passed though the chicane and down the linac where its trajectory was recorded by beam position monitors (BPMs) located in each of the quadrupole magnets.

The transverse wakefield was determined by measuring the changes in the witness bunch vertical deflection that resulted from controlled changes to the drive bunch vertical offset in the structure. To formulate the measurement approach, let $\Delta \mathrm{y}_{\mathrm{d}}$ denote the change in the vertical trajectory of the drive bunch in the structure, parallel to its axis. The resulting change to the witness bunch vertical angular trajectory, $\Delta \theta_{y}$, due to the dipole modes is

$$
\Delta \theta_{y}=A W_{\perp}(t) \Delta y_{d}
$$

where $W_{\perp}(t)$ is the integrated dipole transverse wakefield in the structure at the time, $t$, behind the drive bunch. For convenience, $W_{\perp}$ is normalized in units of the drive bunch offset, drive bunch charge, and structure length. The proportionality factor, $A$, is

$$
A=e^{2} L_{s} I_{d} f_{s} / E_{w}
$$

where $L_{s}$ is the structure length, $I_{d}$ is the drive bunch intensity (i.e., the number of particles in the bunch), $E_{w}$ is the witness bunch energy $(1.2 \mathrm{GeV})$, and $f_{s}$ is a factor that accounts for the averaging of the drive and witness bunch wakefield interaction over the longitudinal distribution of the particles in the bunches (assumed to be Gaussian with a sigma of $1.0 \mathrm{~mm}$ ). As discussed below, the $15.1 \mathrm{GHz}$ central frequency of the lowest dipole band was the dominant wakefield component observed, so the dilution

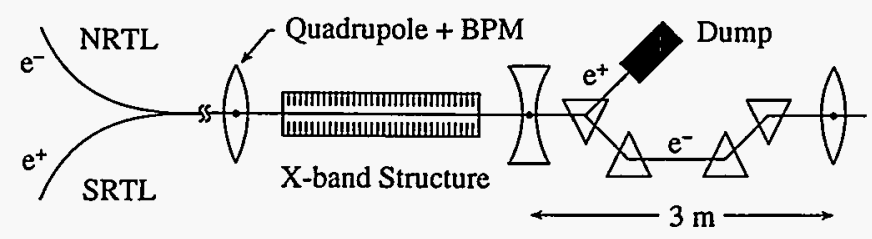

FIG. 1. Layout of ASSET in the SLC.

factor corresponding to this frequency, $\mathrm{f}_{s}=.90$, was used in the calculations.

The witness bunch deflections, $\Delta \theta_{y}$, were computed from betatron oscillation fits to data from 24 BPMs downstream of the chicane, and corrected for incoming orbit jitter using the results from similar fits to data from 17 BPMs in the NRTL. Special care was taken to achieve an accurate measurement of the oscillation parameters. The transport matrix elements needed to do the fits were computed from the vertical oscillations induced by changing the strength of two dipole corrector magnets near the beginning of the NRTL. Computing the elements in this manner incorporates the effects of intra-bunch wakefields, bunch energy and magnet field strength errors, and scale errors of the BPMs in the fit regions. It also makes the wakefield results independent of a common scale error in the two BPMs neighboring the structure which were used to calibrate the bunch steering, and insensitive to the first order difference in these scale errors, which at most should be a few percent.

The positron intensity was measured with a toroid in the SRTL that has a few percent absolute accuracy. The toroid was not recorded pulse-to-pulse but sampled (10 pulse average) every two minutes. The value that was sampled nearest in time to the wakefield measurement was used to compute the wakefield strength. Unfortunately, the positron intensity was unstable for most of the running period, which led to uncertainties in the inferred wakefield strengths that varied from a few percent up to $10 \%$. Also, the average positron intensity decreased from about $2 \times 10^{10}$ to $5 \times 10^{9}$ during the run, and thus reduced the sensitivity to the wakefield proportionally. The electron bunch intensity, however, was fairly stable at about $1 \times 10^{10}$.

To measure the temporal dependence of the wakefield, control of the relative bunch timing was required. Three methods were used, each of which provided a different level of control. The coarsest level was the $8.4 \mathrm{~ns}$ clock period of the SLC timing system. By programmed changes to all pulsed systems affecting the witness bunch, its timing could be shifted in multiples of this period. The change could be made quickly and produced no noticeable differences in the witness bunch orbit upstream of the structure. A finer level of control was achieved by shifting the witness bunch timing in the injector so it entered the North Damping Ring delayed by multiples of the $1.4 \mathrm{~ns}$ ring if period. 


\section{DISCLAIMER}

This report was prepared as an account of work sponsored by an agency of the United States Government. Neither the United States Government nor any agency thereof, nor any of their employees, makes any warranty, express or implied, or assumes any legal liability or responsibility for the accuracy, completeness, or usefulness of any information, apparatus, product, or process disclosed, or represents that its use would not infringe privately owned rights. Reference herein to any specific commercial product, process, or service by trade name, trademark, manufacturer, or otherwise does not necessarily constitute or imply its endorsement, recommendation, or favoring by the United States Government or any agency thereof. The views and opinions of authors expressed herein do not necessarily state or reflect those of the United States Government or any agency thereof. 


\section{DISCLAIMER}

Portions of this document may be illegible in electronic image products. Images are produced from the best available original document. 
Finally, the South Damping Ring if phase control that synchronizes the positron bunch, while stored, to the linac If phase, was used to achieve continuous time changes in the range one S-band period ( $350 \mathrm{ps})$. This phase locking system, which is used for electrons as well, is stable pulseto-pulse at the sub-picosecond level as evidenced by the SLC longitudinal interaction point stability during colliding beam operation.

A two day period was allotted to measure the wakefield in the prototype X-band structure. During this period, data were taken at 18 bunch timing settings that were configured by timing changes of $1.4 \mathrm{~ns}$ and/or $8.4 \mathrm{~ns}$ multiples. At each setting, the continuous bunch timing control was used, and several measurements of the wakefield were made as part of a two step approach to computing the dipole amplitude, $\left|W_{\perp}\right|$. In the first step, the drive bunch offset relative to the structure axis, $y_{d}$, was set to $2.2 \mathrm{~mm}$ using the two neighboring BPMs as an absolute reference. Data were then taken in which the bunch timing was varied over a few oscillation periods of the wakefield. The measured changes in the witness bunch deflection were used to map the local wakefield function, and in particular, to find the time settings corresponding to its peak values, both positive and negative. In the second step, data were taken at a few of the peak time settings in which $y_{d}$ was varied from $-2.0 \mathrm{~mm}$ to $2.0 \mathrm{~mm}$ at three settings of the witness bunch offset, $y_{w}=-0.2,0$, $0.2 \mathrm{~mm}$. Here the $\Delta \theta_{\mathrm{y}}$ results were fit to determine $\left|W_{\perp}\right|$ as well as the integrated quadrupole wakefield strength, for which $\Delta \theta_{y} \propto \Delta y_{d}^{2} \Delta y_{w}$. This two step approach to measuring the amplitude, while much quicker than doing the offset measurements at many time settings, assumes in the first step that the dipole component dominates the total wakefield, and that the structure is well aligned $(\ll 2.2 \mathrm{~mm})$ to the two neighboring BPMs. As measurements were made, the data in fact justified these assumptions and so the acquisition continued in this manner.

Figure 2 shows two examples of measurements made by varying the bunch timing, one near the bunch crossing $(t=0)$, and one where the bunches were about $92 \mathrm{~ns}$ apart. The data points were computed using Eq. (1) where $\Delta y_{d}$ was assumed to equal the absolute drive bunch offset of $2,2 \mathrm{~mm}$. The solid line in Fig. $2 \mathrm{a}$ is a prediction of the short-range wakefield, while that in Fig. $2 b$ is a fit to the data of a sine function with a fixed period. Because of an uncertainty in the calibration of the continuous bunch timing control, the time scale was adjusted so that the period of the observed oscillations, such as seen in Fig. 2b, corresponds to the central dipole mode frequency of $15.1 \mathrm{GHz}$. This same scale factor was used for all fits.

The predicted wakefield in Fig. 2a was obtained by summing the contributions from the synchronous modes that were computed for a periodic structure with an iris radius equal to the average of that in the detuned
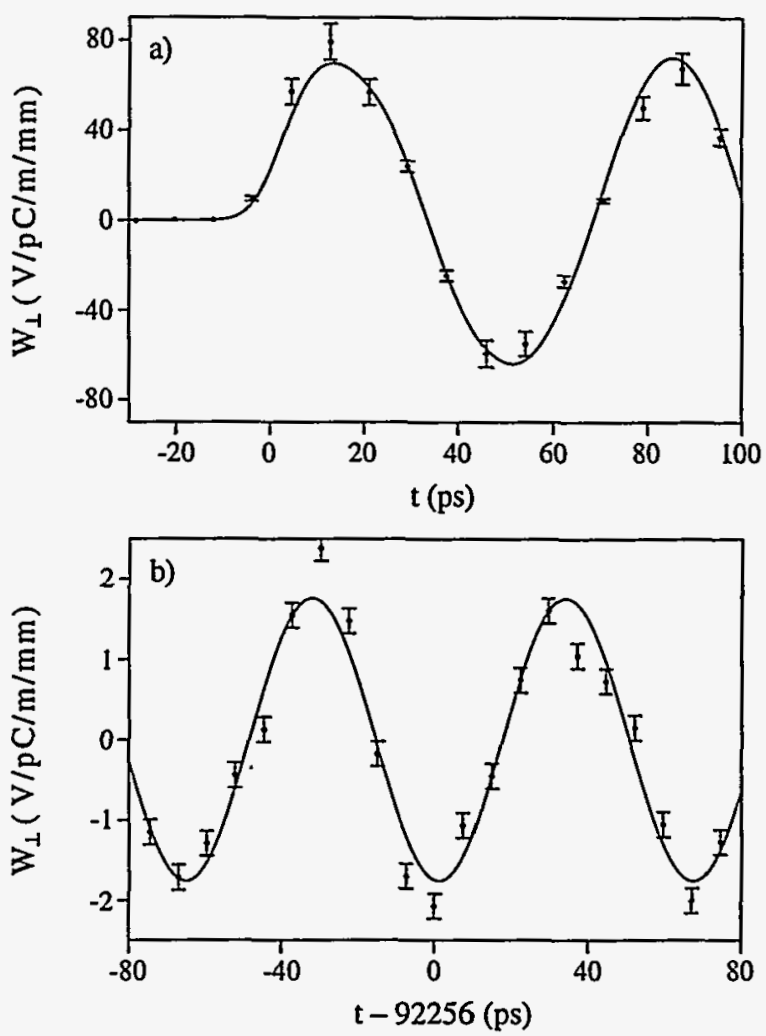

FIG. 2. Dipole wakefield measured (a) near the bunch crossing and (b) at a bunch separation of about $92 \mathrm{~ns}$. The solid lines are described in the text.

structure [8]. Thus the detuning is ignored although its effect is expected to be small on a time scale this short. However, the prediction includes the averaging that results from the non-zero bunch lengths, which further reduces the already small higher band contributions. The $t=0$ point of data was defined as the best match to the prediction (note that the data are causal in that the values at negative times are consistent with zero). The error bars on the data points in this figure include the contribution from the positron intensity jitter, which is generally much larger than that from the $\Delta \theta_{y}$ measurement error.

Figure $2 b$ is typical of the measurements at later times in that there are no apparent contributions from higher frequency modes. The dipole wakefield amplitudes measured from the sine curve fits in fact agree with the results from varying the bunch offsets. This agreement is consistent with the finding of no significant quadrupole contributions in the offset analyses, as was expected. The agreement is also consistent with the $\approx 100 \mu \mathrm{m}$ accuracy to which the structure was aligned to the two neighboring BPMs. A comparison of the witness bunch deflections that were measured at a peak wakefield time setting near $t=0$, with $y_{d}=0$ and with the drive bunch turned off, verify this level of alignment.

The suppression of wakefield amplitude, as evidenced by comparing Figs. $2 a$ and $2 b$, is better illustrated in Fig. 3 , in which all amplitude data are plotted as a 
function of the bunch time separation. Each data point in this figure is typically an average of six measurements, two from repeated bunch time variation measurements, and four from two repeated bunch offset variation measurements at two of the peak wakefield time settings. The error bar on each point however is not the combined errors of the individual measurements, but an estimate based on the variation of the values averaged, and a 5\% systematic uncertainty. The spread in the measured values is correlated with the size of the positron intensity jitter, and is reflected in the $\chi^{2}$ of the fits.

The solid line in Fig. 3a is a prediction of the longrange wakefield based on the equivalent circuit model discussed earlier. One sees that the data are consistent with the initial rapid fall-off in the predicted wakefield amplitude, but that subsequent points up to about $40 \mathrm{~ns}$ are much larger than the predictions, while points at later times are smaller on average by about a factor of two. Part of this disagreement is believed to be due to the effect of the residual errors in the cell cavity diameters from the fabrication process. Measurements of the cells made before the structure was assembled show a Gaussian-like error distribution in the diameters that would produce a $1.5 \times 10^{-4} \mathrm{rms}$ fractional smearing of the synchronous cell dipole mode frequencies. Recomputing the wakefield with different sets of frequency errors, each drawn from a Gaussian distribution with this rms width, produces results
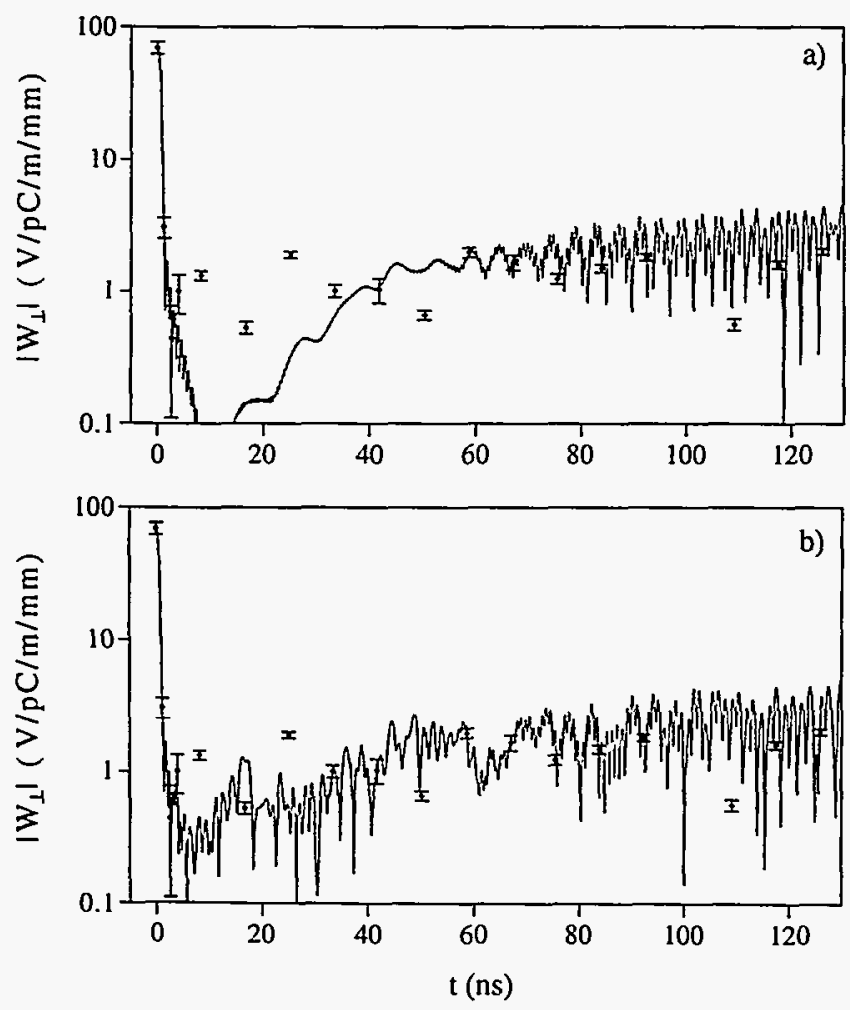

FIG. 3. Dipole wakefield amplitude measurements and prediction a) without cell frequency errors and b) with $1.5 \times 10^{-4} \mathrm{rms}$ fractional frequency errors. closer in size to the $t<40$ ns data. The solid line in Fig. 3b is an example.

At later times, the addition of the frequency errors does not greatly change the results. Here the predictions would better agree with the data if the $\mathrm{Q}$ of the modes used in the calculations were lowered from their assumed value of 6500 to about 4000 . Measurements of the loaded $Q$ of the modes using a network analyzer, however, show no evidence for the smaller values. Cross-plane coupling of the wakefield modes, which would also reduce the measured wakefield, has been ruled out from an analysis of the horizontal orbit data taken during the measurements.

Theoretical work is continuing in an effort to better model the structure. One improvement will be to include the effect of the iris thickness variation in the structure, although it is not expected to change the results significantly. Also, a more realistic treatment of the effect of the input and output couplers with matched loads is being pursued.

In summary, the suppression of the long-range transverse wakefield in a detuned X-band structure has been verified using the ASSET facility in the SLC. The measured wakefield amplitudes are in fair agreement with predictions that include the effect of cell fabrication errors. The short-range wakefield prediction, which is less theoretically uncertain, is in good agreement with the data. These results are an important first step in proving the viability of multi-bunch operation in an NLC-like linac.

The authors thank $M$. Saleski for his considerable work in setting up the bunch timing controls for this project. They also thank F.J. Decker, P. Emma, S. Hartman, $M$. Ross and M. Stanek for their help in data taking. This work was supported by the U.S. Department of Energy under Contract DE-AC03-76F00515.

${ }^{*}$ Visitor from KEK.

[1] K. Thompson et al., Part. Accel., accepted for publication (to appear in vol. 47).

[2] J. Wang et al., in Proceedings of the 17th International Linac Conference, Tsukuba, Japan, Aug. 21-26, 1994 (unpublished).

[3] K. Bane et al., in Proceedings of the 4th European Particle Accelerator Conference, London, England, Jun. 27 - Jul. 1, 1994 (to be published).

[4] K. Bane and R. Gluckstern, Part. Accel. 42, 123 (1993).

[5] N. Kroll et al., in Proceedings of the 17th International Linac Conference (Ref. [2]).

[6] This two beam approach has been used at Argonne, see e.g., J. Simpson et al., Argonne Report No. ANLHEP-CP-86-46 (unpublished).

[7] C. Adolphsen et al., Int. J. Mod. Phys. A, Proc. Suppl. 2B, 870 (1993).

[8] K. Bane, private communication. 